\title{
Phylogenetic analysis of avian paramyxovirus serotype-1 in pigeons in Japan
}

\author{
Masaji MASE ${ }^{1,2) *}$ and Katsushi KANEHIRA ${ }^{1)}$ \\ 1)National Institute of Animal Health, 3-1-5 Kannondai, Tsukuba, Ibaraki 305-0856, Japan \\ ${ }^{2)}$ United Graduate School of Veterinary Sciences, Gifu University, 1-1 Yanagido, Gifu, Gifu 501-1193, Japan
}

(Received 23 December 2014/Accepted 10 March 2015/Published online in J-STAGE 22 March 2015)

ABSTRACT. To understand the epidemiology of Avian paramyxovirus serotype-1 (APMV-1) in pigeons in Japan, phylogenetic analysis was comprehensively conducted based on partial fusion protein gene using isolate from the surveillance of this virus with previously known Japanese pigeon strains. This surveillance was conducted using feces obtained from domestic pigeons collected in 40 prefectures throughout Japan from June 2011 to March 2013. From a total of 1,021 samples, a single virus (APMV1/pigeon/Japan/Kanagawa/2013: JP/Kanagawa$\mathrm{pg} / 2013$ ) was isolated. All Japanese pigeon APMV-1 strains were clustered into a single genetic lineage, which was termed VIb/1 by phylogenetic analysis based on the F gene including the sequence of the cleavage site. These APMV-1 strains were further subdivided into four subgroups identified over 4 separate timeframes: 1984-1995 (group 1), 1995-2000 (group 2), 2001-2007 (group 3) and the novel subgroup isolated in 2013 (group 4). Each subgroup has specific amino acid motifs at a cleavage site of the F protein, namely, ${ }^{112} \mathrm{GRQKR}-\mathrm{F}^{117}$ (except for one strain), ${ }^{112} R R K K R-F^{117},{ }^{112} R R Q K R-F^{117}$ and ${ }^{112} R R Q K R-F^{117}$, respectively. Our data suggest that Japanese APMV-1 strains from pigeons were diverse and reinforced the possibility that there were multiple introduction routes from foreign countries into Japan.

KEY WORDS: avian paramyxovirus serotype-1, phylogeny, pigeon

doi: 10.1292/jvms.14-0684; J. Vet. Med. Sci. 77(8): 919-923, 2015

Newcastle disease (ND) is one of the most serious diseases affecting the poultry industry. This disease is caused by specific strains of avian paramyxovirus serotype-1 (APMV-1), which belongs to the genus Avulavirus in the family Paramyxoviridae [11]. Depending on the virulence of an isolate, Newcastle disease virus (NDV) can be grouped into lentogenic (low virulence), mesogenic (moderately virulent) and velogenic (virulent) pathotypes. Mesogenic and velogenic strains are "virulent" and have been frequently identified as the causative agents of ND outbreaks worldwide [11].

So far, four major panzootics of ND have been recorded [11]. Among them, the third panzootic was caused by the pigeon paramyxovirus type 1 or pigeon type APMV-1. This virus is considered to originate in the Middle East in the late 1970s [3, 7]. By 1981, it reached Europe, and thereafter, the virus rapidly spread throughout the world. Since then, despite various control measures including vaccination, this disease in pigeons remains panzootic $[1,18]$. In Japan, the first outbreak of this disease in pigeons was observed among racing pigeons in September 1983 [15], and thereafter, the outbreaks of this disease in pigeons have been reported for over three decades.

In this study, to further understand the epidemiology of APMV-1 in pigeons in Japan, we conducted the surveillance

\footnotetext{
*Correspondence to: Mase, M., Viral Disease and Epidemiology Research Division,National Institute of Animal Health, National Agriculture and Food Research Organization (NARO), 3-1-5 Kannondai, Tsukuba, Ibaraki 305-0856, Japan.

e-mail: masema@affrc.go.jp

(C)2015 The Japanese Society of Veterinary Science

This is an open-access article distributed under the terms of the Creative Commons Attribution Non-Commercial No Derivatives (by-nc-nd) License $<$ http://creativecommons.org/licenses/by-nc-nd/3.0/>.
}

of this virus using feces from domestic pigeons collected in 40 prefectures throughout Japan from June 2011 to March 2013. We isolated a new virus from these samples and performed comprehensive phylogenetic analysis based on partial fusion protein gene using previously known Japanese pigeon isolates.

\section{MATERIALS AND METHODS}

Surveillance samples: Fecal samples were periodically collected from feral pigeons at each prefecture (every two months in principle) in Japan (except for Aomori, Akita, Miyagi, Aichi, Hyogo, Yamaguchi and Ehime). One sample was principally made by pooling the feces of five birds. The collected fecal samples were placed in screw-cap tubes and stored at $-80^{\circ} \mathrm{C}$ until virus isolation.

Each collected sample was suspended at a concentration of approximately $10 \%$ in Eagle's minimum essential media containing antibiotics. The suspension was centrifuged, and the supernatant was inoculated into the allantoic cavities of 9-12-day-old specific pathogen free (SPF) chicken embryonated eggs. After inoculation, the eggs were incubated at $37^{\circ} \mathrm{C}$ for 6 days unless the embryo died. The inoculated eggs were then chilled to $4^{\circ} \mathrm{C}$, and the allantoic fluids were harvested and tested for hemagglutination activity (HA). For negative samples, two allantoic fluids were pooled and repassaged into two new eggs in order to confirm negativity. Identification of APMV-1 was conducted by the conventional hemagglutination-inhibition (HI) assay with APMV-1 specific antiserum.

Nucleotide sequencing and phylogenetic analysis: Viral RNA was extracted from the infected allantoic fluids using a commercial kit (QIAamp Viral RNA Mini Kit, QIAGEN, Valencia, CA, U.S.A.) according to the manufacturer's in- 
Table 1. APMV-1 strains used in this study

\begin{tabular}{|c|c|c|c|c|}
\hline Virus & Abbreviation & Year & Group & $\begin{array}{l}\text { F protein cleavage site } \\
\text { (Position 112-117) }\end{array}$ \\
\hline APMV1/pigeon/Japan/Ibaraki/84 & JP/Ibaraki-pg/84 & 1984 & 1 & GRQKR-F \\
\hline APMV1/pigeon/Japan/Nagano-8/84 & JP/Nagano-8-pg/84 & 1984 & 1 & GRQKR-F \\
\hline APMV1/pigeon/Japan/FK-1/84 & JP/FK-1-pg/84 & 1984 & 1 & GRQKR-F \\
\hline APMV1/pigeon/Japan/Tochigi/86 & JP/Tochigi-pg/86 & 1986 & 1 & WRQKR-F \\
\hline APMV1/pigeon/Japan/Niigata/88 & JP/Niigata-pg/88 & 1988 & 1 & GRQKR-F \\
\hline APMV1/pigeon/Japan/Kushiro/91 & JP/Kushiro-pg/91 & 1991 & 1 & GRQKR-F \\
\hline APMV1/pigeon/Japan/Tokachi/91 & JP/Tokachi-pg/91 & 1991 & 1 & GRQKR-F \\
\hline APMV1/pigeon/Japan/Kumamoto/95 & JP/Kumamoto-pg/95 & 1995 & 1 & GRQKR-F \\
\hline APMV1/pigeon/Japan/Tochigi/95 & JP/Tochigi-pg/95 & 1995 & 2 & RRKKR-F \\
\hline APMV1/pigeon/Japan/Utsunomiya/95 & JP/Utsunomiya-pg/95 & 1995 & 2 & RRKKR-F \\
\hline APMV1/pigeon/Japan/Shiga/96 & JP/Shiga-pg/96 & 1996 & 2 & RRKKR-F \\
\hline APMV1/pigeon/Japan/Fukushima/96 & JP/Fukushima-pg/96 & 1996 & 2 & RRKKR-F \\
\hline APMV1/pigeon/Japan/Saitama/97 & JP/Saitama-pg/97 & 1997 & 2 & RRKKR-F \\
\hline APMV1/pigeon/Japan/Gunma/2000 & JP/Gunma-pg/2000 & 2000 & 2 & RRKKR-F \\
\hline APMV1/pigeon/Japan/Kumamoto/2001 & JP/Kumamoto-pg/2001 & 2001 & 3 & RRQKR-F \\
\hline APMV1/pigeon/Japan/Hiroshima/2002 & JP/Hiroshima-pg/2002 & 2002 & 3 & RRQKR-F \\
\hline APMV1/pigeon/Japan/Mie/2002 & JP/Mie-pg/2002 & 2002 & 3 & RRQKR-F \\
\hline APMV1/pigeon/Japan/Shizuoka/2002 & JP/Shizuoka-pg/2002 & 2002 & 3 & RRQKR-F \\
\hline APMV1/pigeon/Japan/Saitama-1/2003 & JP/Saitama-pg-1/2003 & 2003 & 3 & RRQKR-F \\
\hline APMV1/pigeon/Japan/Saitama-2/2003 & JP/Saitama-pg-2/2003 & 2003 & 3 & RRQKR-F \\
\hline APMV1/pigeon/Japan/Tokyo/2006 & JP/Tokyo-pg/2006 & 2006 & 3 & RRQKR-F \\
\hline APMV1/pigeon/Japan/Niigata/2007 & JP/Niigata-pg/2007 & 2007 & 3 & RRQKR-F \\
\hline APMV1/pigeon/Japan/Kanagawa/2013 & JP/Kanagawa-pg/2013 & 2013 & 4 & RRQKR-F \\
\hline
\end{tabular}

structions. Reverse transcription, PCR amplification and sequencing were performed as described previously [9]. Briefly, after reverse transcription with SuperScript ${ }^{\mathrm{TM}}$ III (Life Technologies, Gaithersburg, MD, U.S.A.) using random 9 mers, PCR was performed to amplify cDNAs using Takara ExTaq (Takara, Tokyo, Japan). For PCR amplification, 35 cycles were performed at $94^{\circ} \mathrm{C}$ for $30 \mathrm{sec}, 50^{\circ} \mathrm{C}$ for $30 \mathrm{sec}$ and $72^{\circ} \mathrm{C}$ for $30 \mathrm{sec}$. The primer sequence and location were as follows: NDV-F2 (4235 5'-TGGAGCCAAACCGCGCACCTGCGG-3' 4258; and NDV-R2 (5000 5'-GGAGGATGTTGGCAGCAT-3' 4983. The sequences were numbered according to previously published sequence (GenBank Acc. No.AF309418). The sequences amplified by these primers included the cleavage site of the $\mathrm{F}$ protein and the region to construct a phylogenetic tree for genotyping the virus. PCR amplification and sequencing were performed as previously described [9]. A phylogenetic tree was constructed by the neighbor-joining method [12] in MEGA6 [16].

For the comprehensive analysis of APMV-1 in pigeons in Japan, we used the new isolate obtained in this surveillance study and compared it with the Japanese APMV-1 strains isolated from pigeons from 1984 to 2007 (Table 1).

Pathogenicity test using intracerebral pathogenicity index: We compared the pathogenicity of representative strains of each group by the intracerebral pathogenicity index (ICPI) test according to the Office International des Epizooties (OIE) procedure [11]. Briefly, one-day-old SPF chickens were intra-cerebrally inoculated with $0.05 \mathrm{~m} l$ of a 1:10 dilution of infective allantoic fluid. The chicks were monitored during an 8-day observation period and scored as normal (score 0 ), sick or paralyzed (score 1 ) and dead (score 2). Total scores were determined, and the mean daily scores were calculated to obtain the ICPI.

Hemagglutination inhibition test: To understand the differences in antigenicity among pigeon APMV-1 strains, the $\mathrm{HI}$ test using four antisera against APMV-1 was performed using representative strains of each group as previously described [6].

\section{RESULTS}

Isolation and characterization of the isolate: A total of 1,021 samples were collected during the study period. One APMV-1 virus was isolated from the sample collected at Kanagawa prefecture (sampling site: Sagamihara city) in February 2013. We designated this isolate as APMV1/ pigeon/Japan/Kanagawa/2013 and abbreviated it as JP/ Kanagawa-pg/2013. As a result of the direct sequencing of the obtained PCR product, the isolate showed the deduced amino acid sequence ${ }^{112} \mathrm{RRQKR}-\mathrm{F}^{117}$ at the F0 protein cleavage site, which was identical to a virulent motif (Table 1).

Comprehensive phylogenetic analysis of APMV-1 in pigeons in Japan: Using phylogenetic analysis based on the $\mathrm{F}$ gene including the sequence of the cleavage site, all APMV1 strains isolated from pigeons including JP/Kanagawa$\mathrm{pg} / 2013$ were clustered into a single genetic lineage, which 
was termed $\mathrm{VIb} / 1$ within genotype VI: this is synonymous with lineage $4 \mathrm{~b}[1,18]$ (Fig. 1). These APMV-1 strains were further subdivided into four subgroups identified over 4 separate timeframes: 1984-1995 (group 1), 1995-2000 (group 2), 2001-2007 (group 3) and the novel subgroup isolated in 2013 (group 4). The group 1 strains were clustered together with the strains belonging to the genetic lineage $\mathrm{VIb} / 1$ early European (EU/ea) group [18]. There was at least 98.7\% identity at the nucleotide level between the group1 strains.

The group 2 APMV-1 strains were clustered together with the strains belonging to the genetic lineage VIb/1-recent European 1 (EU/re1) group [18]. Among them, the JP/Tochigipg/95 strain shares $100 \%$ nucleotide identity with a German APMV-1 strain (DE-48/92) [18], which was also isolated from a pigeon. Furthermore, the JP/Shiga-pg/96 strain also shares $100 \%$ nucleotide identity with a German strain (DE57/95) [18], which was also isolated from pigeons. The group 3 APMV-1 strains were clustered together with the strains belonging to the genetic lineage VIb/1-recent European 2 (EU/re2) group [18]. These strains were further subdivided into two groups that were composed of strains in eastern Japan and western Japan, respectively (Fig. 1). Interestingly, the phylogenetic analysis indicated that the APMV-1 strains isolated in western Japan (Kumamoto, Hiroshima and Mie) were clustered together with many Chinese strains isolated from pigeons. On the other hand, the APMV-1 strains isolated in eastern Japan (Saitama, Tokyo, Shizuoka and Niigata) were clustered together with the viruses isolated in European countries and the U.A.E. (Fig. 1).

The group 4 APMV-1 strain was clustered together with the strains belonging to the genetic lineage $\mathrm{VIb} / 1$, a recent European pigeon APMV-1 group mainly prevalent Belgium and Macedonia (Fig. 1).

Each group in Japan has specific amino acid motifs at the cleavage site of the F protein, namely, ${ }^{112} \mathrm{GRQKR}-$ $\mathrm{F}^{117}$ (except for one strain) (group 1), ${ }^{112} \mathrm{RRKKR}-\mathrm{F}^{117}$ (group 2), ${ }^{112}$ RRQKR-F ${ }^{117}$ (group 3) and ${ }^{112}$ RRQKR-F ${ }^{117}$ (group 4), respectively.

Pathogenicity by the ICPI test: The ICPI of these strains ranged from 0.8 to 1.1 (Table 2) and were all proven to be pathogenic types, when assessed using criteria of OIE. These indices were slightly lower than that of the first pandemic virus in pigeons isolated during 1983-1984 and suggest that numbers of basal amino acids were not associated with virulence in APMV-1 strains from pigeons as previously reported [5].

Antigenic analysis by the HI test: As shown in Table 2, no major antigenic differences were observed among these strains.

\section{DISCUSSION}

Outbreaks of ND in pigeons are still being reported across the world, including Japan [1]. The origin of the panzootic in pigeons, which was defined as the third panzootic, appeared to be from the Middle East during the late 1970s [3, 7]. This panzootic peaked during the early 1980s, which lead to a number of outbreaks of this disease in poultry and highlighted the importance of these pigeon-derived viruses in posing a real and continuous threat to the poultry industry $[2,5]$. In Japan, outbreaks of this disease in pigeons were prevalent in the mid-1980s [15]. Thereafter, these have been sporadic reports of cases and outbreaks of this disease in pigeons. Although surveillance for virus in feral waterfowl has been previously conducted $[10,13,14,19]$, it has not been comprehensively performed in feral pigeons. Previously, Teske et al. [17] conducted surveillance for the virus in racing pigeons. In their report, although they could detect viral RNA by real-time PCR assay, the virus was not isolated using embryonated eggs.

In this surveillance study, a single virus was successfully isolated from 1,021 samples during a 2-year period (isolation rate approximately $0.1 \%$ ). This isolation rate is lower than that (approximately 1\%) in migratory waterfowls by our recent report [10]. To our knowledge, this study is the first description of APMV-1 isolation from the feces collected from feral pigeons. This isolate was classified into genotype VI and was clustered only with viruses previously isolated from pigeons, suggesting that they were mainly transmitted among pigeons. To date, this type has mostly been isolated from pigeons in Japan [8,9] and has rarely been isolated from poultry. However, the isolated virus in our study was judged as a virulent type, suggesting that it posed a threat to the poultry industry [2]. Indeed, JP/Kanagawa-pg/2013 isolate was genetically close to virus isolated from chicken in Macedonia (Fig. 1).

All Japanese pigeon APMV-1 strains, including isolates in this surveillance study, were clustered into a single genetic lineage, which was termed VIb/1 [18] within genotype VI. These strains were further subdivided into four distinct subgroups (groups 1-4) of lineage VIb/1. Among them, the virus isolated in this surveillance was categorized as group 4 , which was different from those previoulsy isolated (groups 1-3). Additionally, each subgroup has specific amino acid motifs at the cleavage site of the $\mathrm{F}$ protein. These results suggest the possibility of multiple introduction routes from foreign countries into Japan. For example, the trading of pigeons from foreign countries, such as Belgium, has been continuing every year according to the annual report of the quarantine service of the Ministry of Health, Labour and Welfare of Japan. We speculate that this is one of the introduction routes for pigeon APMV-1 into Japan. To prevent the introduction of pigeon APMV-1 into Japan, it might be necessary to strengthen such quarantine system.

The group 3 strains isolated in western Japan from 2001 to 2002 were genetically related to many Chinese strains [4]. Among them, the W4 isolate has been suggested to be associated with waterfowl migration. This suggested that the virus is introduced from migrating birds. In order to further understand the epidemiology of APMV-1 in Japan and the role of pigeons as a vehicle of transmission, surveillance should be continued over the next few years.

ACKNOWLEDGMENTS. This work was supported by a Grant-in-Aid from the Regulatory Science Project of the Ministry of Agriculture, Forestry and Fisheries of Japan. 


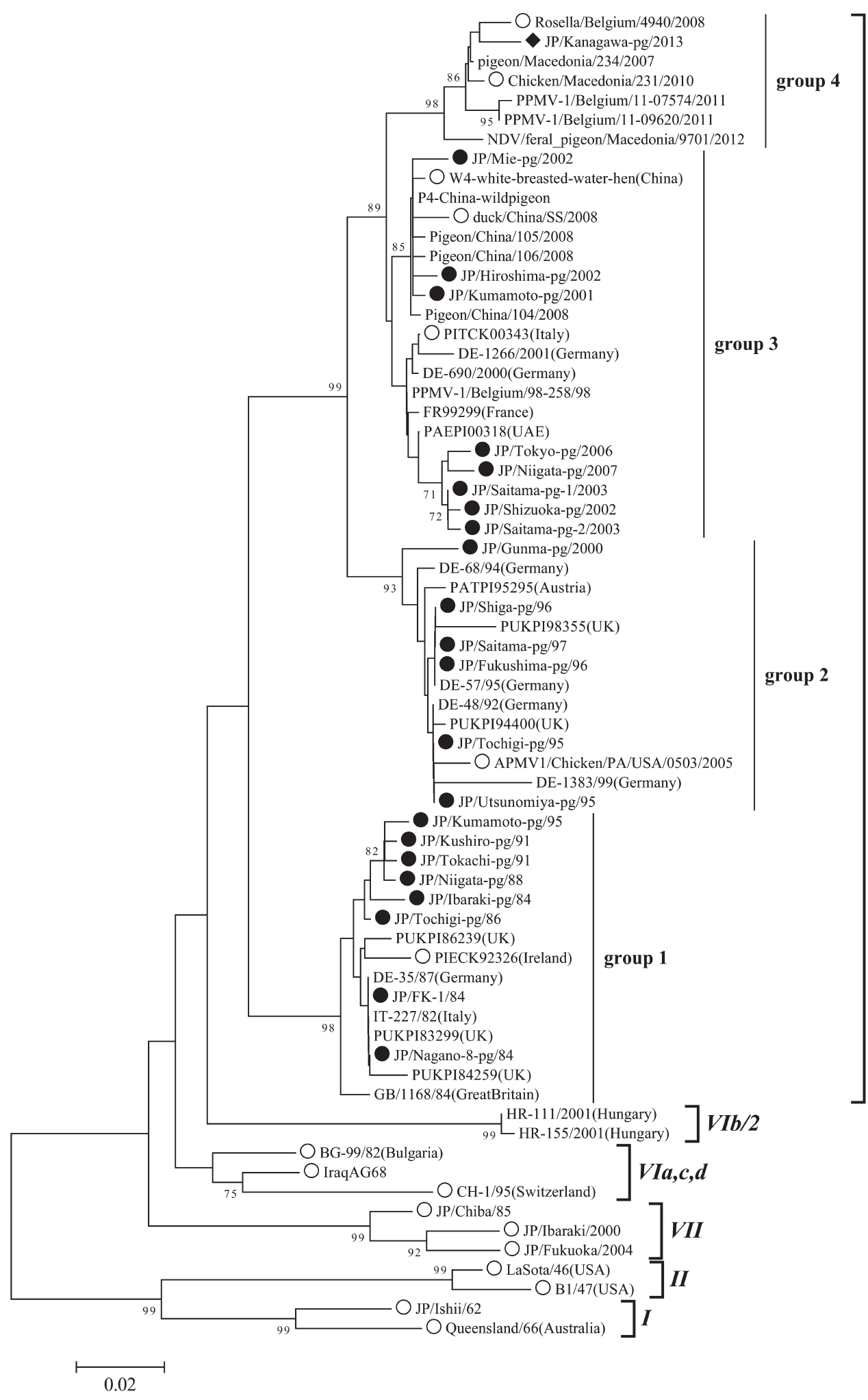

Fig. 1. Phylogenetic tree of the APMV-1 isolates mainly isolated from pigeons based on nucleotide sequences from a portion (nt 47-420) of the F gene. The horizontal distances are proportional to the minimum number of nucleotide differences required to join nodes and sequences. The new isolate in this surveillance is shown by black rhombus. Japanese NDV strains previously isolated from pigeons are shown by black circle. The viruses that were not isolated from pigeon were shown by white circle. The tree was generated using the neighbor-joining algorithm, and alignments were bootstrapped 1,000 times. Bootstrap values $>70$ are shown. 
Table 2. Results of the ICPI and HI tests among representative APMV-1 strains isolated from pigeons

\begin{tabular}{lcccccc}
\hline \multirow{2}{*}{ Viruses } & \multirow{2}{*}{ Group } & \multirow{2}{*}{ ICPI } & \multicolumn{4}{c}{ Antiserum against } \\
\cline { 4 - 7 } & & & B1/47 & JP/Sato/30 & JP/Shizuoka/85 & JP/Nagano-8-pg/84 \\
\hline JP/Kumamoto-pg/95 & 1 & 1.1 & 32 & 512 & 1024 & 256 \\
JP/Tochigi-pg/95 & 2 & 1.0 & 64 & 512 & 1024 & 256 \\
JP/Kumamoto-pg/2001 & 3 & 1.1 & 32 & 512 & 512 & 128 \\
JP/Saitama-pg-2/2003 & 3 & 0.8 & 16 & 256 & 512 & 64 \\
JP/Kanagawa-pg/2013 & 4 & 1.1 & 32 & 512 & 512 & 64 \\
\hline
\end{tabular}

We thank the staffs in each prefecture that collected the fecal samples. The authors wish to thank Mr. T. Inoue for his technical assistance in this study.

\section{REFERENCES}

1. Aldous, E. W., Fuller, C. M., Mynn, J. K. and Alexander, D. J. 2004. A molecular epidemiological investigation of isolates of the variant avian paramyxovirus type 1 virus (PPMV-1) responsible for the 1978 to present panzootic in pigeons. Avian Pathol. 33: 258-269. [Medline] [CrossRef]

2. Alexander, D. J., Russell, P. H. and Collins, M. S. 1984. Paramyxovirus type 1 infections of racing pigeons: 1 characterisation of isolated viruses. Vet. Rec. 114: 444-446. [Medline] [CrossRef]

3. Biancifiori, F. and Fioroni, A. 1983. An occurrence of Newcastle disease in pigeons: virological and serological studies on the isolates. Comp. Immunol. Microbiol. Infect. Dis. 6: 247-252. [Medline] [CrossRef]

4. Cai, S., Li, J., Wong, M. T., Jiao, P., Fan, H., Liu, D., Liao, M., Jiang, J., Shi, M., Lam, T. T., Ren, T. and Leung, F. C. 2011. Genetic characterization and evolutionary analysis of 4 Newcastle disease virus isolate full genomes from waterbirds in South China during 2003-2007. Vet. Microbiol. 152: 46-54. [Medline] [CrossRef]

5. Dortmans, J. C., Rottier, P. J., Koch, G. and Peeters, B. P. 2011. Passaging of a Newcastle disease virus pigeon variant in chickens results in selection of viruses with mutations in the polymerase complex enhancing virus replication and virulence. J. Gen. Virol. 92: 336-345. [Medline] [CrossRef]

6. Imai, K., Tezuka, H. and Yuasa, N. 1986. Efficacy of Newcastle disease (ND) vaccine (B1 strain) against ND viruses isolated in 1985 in Japan. J. Jpn. Soc. Poult. Dis. 22: 17-21 (in Japanese).

7. Kaleta, E. F., Alexander, D. J. and Russell, P. H. 1985. The first isolation of the avian PMV-1 virus responsible for the current panzootic in pigeons? Avian Pathol. 14: 553-557. [Medline] [CrossRef]

8. Mase, M., Imai, K., Sanada, Y., Sanada, N., Yuasa, N., Imada, T., Tsukamoto, K. and Yamaguchi, S. 2002. Phylogenetic analysis of Newcastle disease virus genotypes isolated in Japan. J. Clin. Microbiol. 40: 3826-3830. [Medline] [CrossRef]
9. Mase, M., Inoue, T. and Imada, T. 2009. Genotyping of Newcastle disease viruses isolated from 2001 to 2007 in Japan. J. Vet. Med. Sci. 71: 1101-1104. [Medline] [CrossRef]

10. Mase, M. and Kanehira, K. 2014. Surveillance of avian paramyxovirus serotype-1 in migratory waterfowls in Japan between 2011 and 2013. J. Vet. Med. Sci. 77: 381-385. [Medline]

11. Miller, P. J. and Koch, G. 2013. Newcastle disease. pp. 89-107. In: Diseases of Poultry, 13th ed. (Swayne, D. E., Glisson, J. R., McDougald, L. R., Nolan, L. K., Suarez, D. L. and Nair, V. eds.). Wiley-Blackwell, Ames.

12. Saitou, N. and Nei, M. 1987. The neighbor-joining method: a new method for reconstructing phylogenetic trees. Mol. Biol. Evol. 4: 406-425. [Medline]

13. Sakai, K., Sakabe, G., Tani, O., Watanabe, Y., Jahangir, A., Nakamura, M. and Takehara, K. 2007. Characterization of Newcastle disease virus isolated from northern pintail (Anas acuta) in Japan. J. Vet. Med. Sci. 69: 1307-1311. [Medline] [CrossRef]

14. Shengqing, Y., Shinya, K., Otsuki, K., Ito, H. and Ito, T. 2002. Isolation of myxoviruses from migratory waterfowls in San-in district, western Japan in winters of 1997-2000. J. Vet. Med. Sci. 64: 1049-1052. [Medline] [CrossRef]

15. Shirai, J., Tsukamoto, K. and Hihara, H. 1986. Newcastle disease viruses isolated from racing pigeons in Japan. J. Vet. Med. Sci. 48: 1091-1095. [Medline] [CrossRef]

16. Tamura, K., Stecher, G., Peterson, D., Filipski, A. and Kumar, S. 2013. MEGA6: Molecular Evolutionary Genetics Analysis version 6.0. Mol. Biol. Evol. 30: 2725-2729. [Medline] [CrossRef]

17. Teske, L., Ryll, M. and Rautenschlein, S. 2013. Epidemiological investigations on the role of clinically healthy racing pigeons as a reservoir for avian paramyxovirus-1 and avian influenza virus. Avian Pathol. 42: 557-565. [Medline] [CrossRef]

18. Ujvári, D., Wehmann, E., Kaleta, E. F., Werner, O., Savić, V., Nagy, E., Czifra, G. and Lomniczi, B. 2003. Phylogenetic analysis reveals extensive evolution of avian paramyxovirus type 1 strains of pigeons (Columba livia) and suggests multiple species transmission. Virus Res. 96: 63-73. [Medline] [CrossRef]

19. Umali, D. V., Ito, H., Katoh, H. and Ito, T. 2014. Surveillance of avian paramyxovirus in migratory waterfowls in the San-in region of western Japan from 2006 to 2012. J. Vet. Med. Sci. 76: 423-430. [Medline] [CrossRef] 